\title{
Polarized M2 macrophages in dogs with visceral leishmaniasis
}

\section{Pamela Rodrigues Reina Moreira ${ }^{a, *}$, Filipe Santos Fernando ${ }^{b}$, Hélio José Montassier $^{c}$, Marcos Rogério André ${ }^{c}$, Rosemeri de Oliveira Vasconcelos ${ }^{c}$}

\author{
a FCAV/UNESP (Faculdade de Ciências Agrárias and Veterinárias, Universidade Estadual Paulista), Jaboticabal, São Paulo, Brazil \\ b Postgraduate Veterinary Medicine Program, FCAV/UNESP, Jaboticabal, São Paulo, Brazil

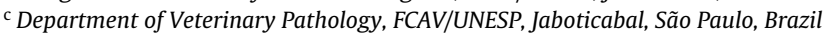

\section{A R T I C L E I N F O}

\section{Article history:}

Received 22 January 2016

Received in revised form 17 June 2016

Accepted 24 June 2016

\section{Keywords:}

Phenotype phagocytes

Dogs

Leishmania infantum

\begin{abstract}
A B S T R A C T
The objective of the present study was to analyze the skin (nasal surface and ear regions), lymph nodes (popliteal and pre-scapular), spleen and liver of dogs with visceral leishmaniasis (VL), in order to investigate the relationship between the parasite load measured as DNA copy number of Alpha gene of DNA polymerase of Leishmania infantum by quantitative PCR and the number of M2 macrophages by immunohistochemistry. A set of 29 naturally infected dogs from an endemic area for VL were sampled and another set of six dogs negative for VL and from a non-endemic area were analyzed as the control group (C). The spleen presented the highest number of Leishmania DNA copies, with significant differences between the groups G1 and G2 (with and without skin lesions, respectively). The M2 phenotype immunostaining predominated among the macrophages in granulomas and inflammatory infiltrates of samples from the skin, lymph nodes and spleens examined. The presence of M2 macrophages in dogs from infected group differed significantly from the control group, in all organs analyzed, excepted liver. The highest proportion of M2 macrophages coincided with the highest parasitism loads found in more susceptible organs of VL dogs, even in the skin, considered a more resistant organ, while the liver showed low parasitism load and low immunostaining for M2 macrophages with no significant differences between infected and negative groups. It was concluded that the predominance of M2 phenotype in VL dogs favored the multiplication of Leishmania infantum in organs of dogs that are more susceptible to Leishmania infection, as skin, lymph nodes and spleen.
\end{abstract}

(C) 2016 Elsevier B.V. All rights reserved.

\section{Introduction}

The role of dogs as the main reservoir of visceral leishmaniasis has led to an increased interest in the immune responses and in Leishmania antigens that are implicated, or in protective cellular immunity against canine visceral leishmaniasis, or conversely involved in immunopathogenesis that lead to the progression of this disease. The main effector mechanism involved in protective immune response of dogs infected with Leishmania (L.) infantum is the activation of macrophages by IFN- $\gamma$ and TNF- $\alpha$, secreted by a predominant response of Th1 cells, which lead to the death of intracellular Leishmania amastigotes. This protective immune response confers apparent resistance to visceral leishmaniasis. However, Leishmania produced a number of factors able to subvert the host immunity, specially by reducing macrophage microbicidal mecha-

\footnotetext{
* Corresponding author at: Via de Acesso Prof. Paulo Donato Castellane s/n, 14884900, Jaboticabal, São Paulo, Brazil.

E-mail address: pamela_rreina@yahoo.com.br (P.R.R. Moreira).
}

nisms. This macrophage suppressive activity of Leishmania seems to depend on an increasing in Th2 function and the secretion of IL-4 and IL-10 and, though the role of these cytokines in symptomatic animals is still controversial, there is an increasing evidence for a correlation of these cytokines with progressive disease (Barbiéri, 2006; Chan et al., 2012).

In human leishmaniasis, Th2 cytokines (IL-4 in cutaneous leishmaniasis; IL-10 and TGF- $\beta$ in visceral leishmaniasis) have been correlated with increased disease susceptibility and its progression was also associate to the induction of the M2 macrophage phenotype, which seems to exert a predominant immunosuppressive action (Cecílio et al., 2014). Muraille et al. (2014) also reported that Leishmania sp. promotes M2 activation, as a virulence and evasion strategies.

In addition, M2 polarized macrophages have been associated with the dysfunction of protective cell-mediated adaptive immune responses in cancer and human leishmaniasis (Edin et al., 2012; Chan et al., 2012). Thus, at the beginning of human leishmaniasis an increased mRNA expression of classical M2 markers and the presence of some receptors/antigens, such as scavenger recep- 
tor/CD 163 in monocytes and macrophages from tissue lesions, has indicated that this macrophage phenotype might contribute to the disease chronicity (Chan et al., 2012). In fact, the CD163 is a useful marker for the identification of M2 macrophages by immunohistochemistry using an anti-CD163 monoclonal antibody raised against human macrophages that cross-reacts with macrophage subpopulations in different animal species including dogs (Komohara et al., 2006; Yamate et al., 2000).

However, there are few studies describing the responses to injuries in different organs of dogs infected by Leishmania infantum and the role of M2 macrophages in the course of Leishmaniasis in these animals. Therefore, the objective of the present study was to analyze lymph nodes (popliteal and pre-scapular), liver, spleen and skin (nasal surface and ear) of dogs naturally infected by Leishmania infantum, in order to investigate the relationship between the presence of the parasite and the macrophages from M2 phenotype.

\section{Material and methods}

\subsection{Material collection}

The dogs investigated in this study were originated from the Zoonosis Control Center in Araçatuba, (São Paulo State, Brazil), a region that is endemic for VL (Moreira et al., 2010; Moreira et al., 2013). Twenty-nine Leishmania infantum - infected dogs were used, without preference for age, breed or gender. The animals were euthanized using an intravenous (IV) overdose of barbiturate, followed by IV administration of potassium chloride (decree number 51.838 of the Brazilian Ministry of Health and Resolution number 714 , of June 20, 2002, of the Federal Veterinary Medicine Council). The necropsy of the dogs was performed immediately after their death. The control group consisted of six dogs from the routine of the Department of Veterinary Pathology Jaboticabal-SP, Brazil, a non-endemic area for VL (Oliveira et al., 2008). Infected dogs and control dogs were selected, following confirmation or not of disease respectively, by RIFI and ELISA.

Fragments of lymph nodes (popliteal and pre-scapular), liver, spleen and skin (nasal surface and ear) were collected. For Immunohistochemical analyses the fragments were fixed in $10 \%$ formalin solution and for the RT-qPCR technique, a fragment of each organ was collected and stored in liquid nitrogen at $-196^{\circ} \mathrm{C}$ until the analysis by this technique.

\subsection{Immunohistochemical analysis}

To perform the immunohistochemical technique, the fragments were fixed in $10 \%$ formalin solution, buffered with 0.15 molar phosphate at pH 7.2 and, after $24 \mathrm{~h}$ of fixing, they were dehydrated, processed, embedded in paraffin and cut into sections of $5 \mu \mathrm{m}$ of thickness. Antigenic recovery was performed by heat using a Pascal pressure chamber (Dako Cytomation, Carpinteria, USA) and a $10 \mathrm{mM}$ sodium citrate buffer solution ( $\mathrm{pH} \mathrm{6.0).} \mathrm{To} \mathrm{double}$ block nonspecific reactions a protein block solution (Dako Cytomation, reference X0909) was added to $3 \%$ of skim powdered milk solution and used over the tissue sections. Following this, the primary antibody (anti-CD163, mouse monoclonal, Spring, reference E18684) was added at 1:300 dilution and incubated for $18 \mathrm{~h}$ at $4{ }^{\circ} \mathrm{C}$. The blocking of endogenous peroxidase was performed using $12 \%$ methanol and hydrogen peroxide solution. Streptavidinbiotin-peroxidase complex (LSAB kit, Dako Cytomation, reference K0690-1) was used as detector system following the instructions of manufacturer and in the last step, DAB substrate-chromogen solution (3,3-diaminobenzidine; Dako Cytomation, reference K3468-1) was used for the color development.
Negative controls consisted of immunohistochemical reactions performed as described above, except that the primary antibody was omitted and only the antibody diluent (Dako Cytomation, reference S302283-2) was used. Positive controls were produced using tissues suggested by the manufacturer of anti-CD163 antibody (Spring, reference E18684).

To determine the number of immunostained cells, five microscope fields were analyzed (Nikon Eclipse E200) with a 40x objective lens (Moreira et al., 2010), which presented an area of approximately $0.19625 \mathrm{~mm} 2$. From the values obtained in these fields, an average number of immunostained cells was determined for each group (Leishmania infected and negative controls).

\subsection{Quantification of Leishmania infantum DNA using $q P C R$}

Samples of spleen, liver, skin (ear and nasal surface) and popliteal and pre-scapular lymph nodes were subjected to total DNA extraction, using the QIAamp DNA Mini Kit (Qiagen, USA), following the manufacturer instructions.

Real-time qPCR using the SYBR Green I marker was used for absolute quantification. Briefly, 5.0 $\mu$ L of DNA extracted were added to a mixture containing $10.0 \mu \mathrm{L}$ of the Kapa Sybr ${ }^{\circledR}$ Fast qPCR Master Mix (2X) (Kapa Biosystems, USA) and 10 pmol of each forward and reverse primers specific for a region in Alpha gene of DNA polymerase of Leishmania infantum (Bretagne et al., 2001) in a final volume of $20 \mu \mathrm{L}$. PCR conditions were as follows: an initial denaturation step at $95^{\circ} \mathrm{C}$ for $3 \mathrm{~min}$, followed by 40 cycles at $95^{\circ} \mathrm{C}$ for $3 \mathrm{~s}, 30 \mathrm{~s}$ for $60^{\circ} \mathrm{C}$ and $72^{\circ} \mathrm{C}$ for $3 \mathrm{~s}$. All the samples were tested in duplicates.

A standard curve was prepared for absolute DNA quantification of a $90 \mathrm{bp}$ fragment of the alpha gene of DNA polymerase (GenBank, access number: AF009147) inserted into the pIDTSmart vector (Integrated DNA Technologies, USA). The plasmid DNA was quantified by spectrophotometer and converted to the number of moles using the Molbio.ru program (www.molgen.mpg.de/ *soldatov/protocols/scripts/01_07.html) and submitted to ten-fold serial dilutions, and each dilution from $10^{-1}$ to $10^{-8}$ was processed by SYBR Green I real-time PCR.

The threshold cycle (CT) values resulting from the real-time PCR from each dilution were used to calculate and determine a linear regression from the logarithmic value of the number of moles of plasmid DNA and used to estimate the number of DNA copies of the Alpha gene of DNA polymerase of Leishmania present in the tissue sample.

\subsection{Statistical analysis}

Statistical analysis was carried out using the nonparametric Mann Whitney test, with comparisons between the groups of dogs (Leishmania-infected and control) to the number of M2 macrophages and between dogs with skin lesions and without skin lesions to the parasite load (number of DNA copies of Leishmania). The correlations between parasite load and number of M2 macrophages in each organ were determined using Spearman's correlation coefficient. The GraphPad Prism statistical software (version 5.00, 2007) was used for all the analyses, and differences were taken to be significant when $\mathrm{P}<0.05$.

\section{Results}

The M2 phenotype was found in macrophages of granulomas with or without parasites and in macrophages of inflammatory infiltrates, in all the organs analyzed (Fig. 1). Within each organ, significant differences were found between the experimental groups. The dogs of the infected group had higher medians. The liver was 


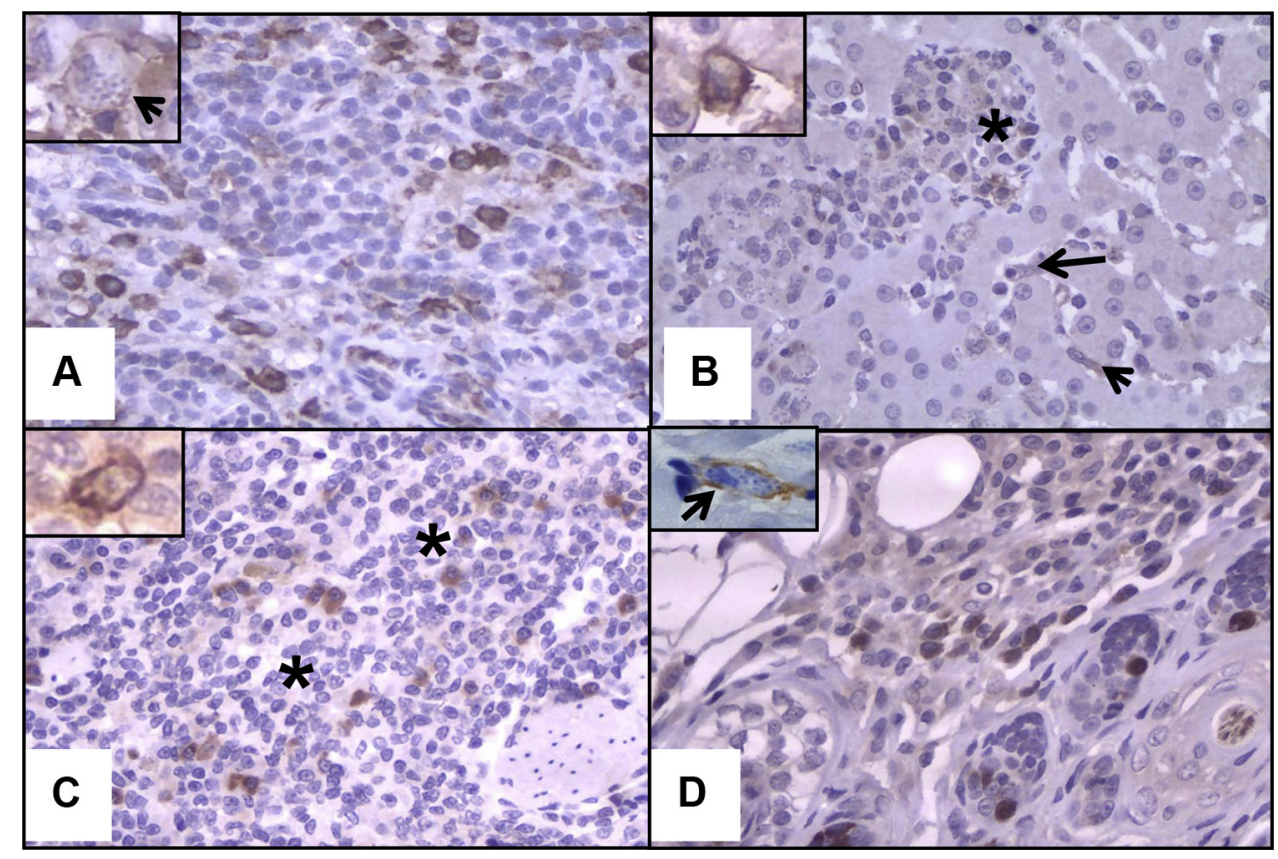

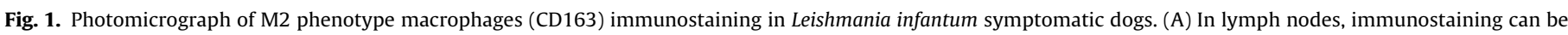

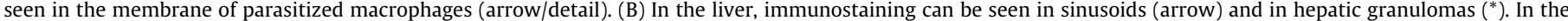

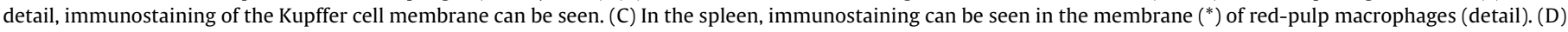

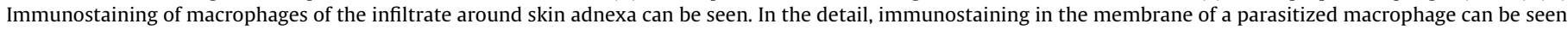
(arrow). Polymer complex bonded to peroxidase ( $40 \mathrm{x}$ objective lens for main figures and also for details).

the organ that not differed between Leishmania-infected and negative control groups (Fig. 2 and Table 1).

The dogs with skin lesions presented higher Leishmania infantum DNA copies (Table 1). When the Leishmania DNA loads from different organs of groups G1 (with skin lesions) and G2 (without skin lesions) were compared, only the spleen $(\mathrm{P}=0.0010)$, popliteal $(\mathrm{P}=0.0037)$ and pre-scapular lymph nodes $(\mathrm{P}=0.0049)$ showed significant difference. The liver had low parasitism in comparison with the other organs.

The correlation between the number of DNA copies of Leishmania-DNA and the number of M2 macrophage were significant only in the popliteal $(\mathrm{P}=0.015 ; \mathrm{r}=0.449)$ and prescapular $(P=0.003 ; r=0.525)$ lymph nodes (Fig. 3 ).

\section{Discussion}

In the present study, the spleen from Leishmania infected dogs was the organ showing the highest level of parasitism which is significant different from the group of non-infected dogs, thus suggesting that it would be more susceptible to multiplication of this parasite. Conversely, the liver seems to be a less favorable environment for the parasite, because it presented low parasitism. These results agreed with the findings of Sanchez et al. (2004) and Lima et al. (2007), demonstrating that the liver had similar low levels of Leishmania parasitism. Furthermore, Reis et al. (2009) described distinct parasite density patterns in the tissues of dogs infected by L. infantum and demonstrated that the skin and spleen were

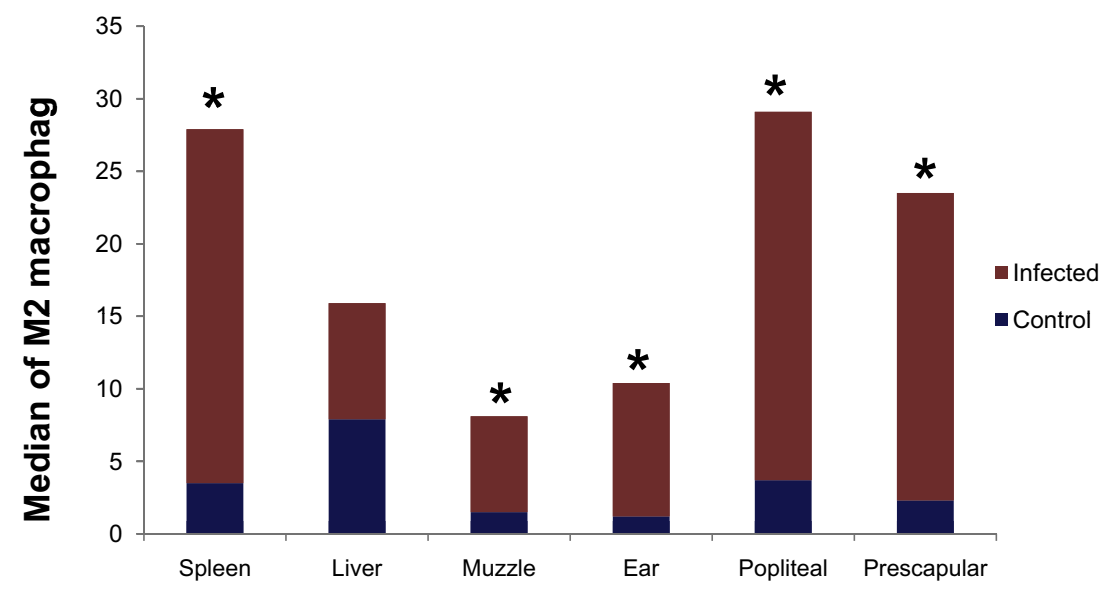

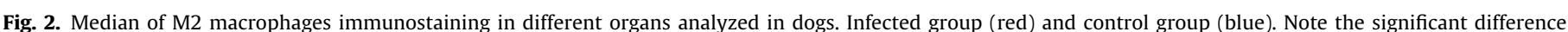

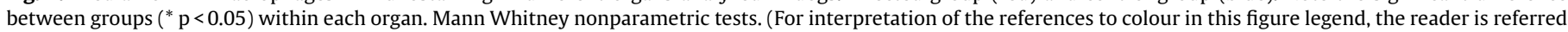
to the web version of this article.) 
Table 1

Medians of M2 macrophages and of the number of Leishmania infantum DNA copies, compared between the groups and within each organ, among the control and VL dogs.

\begin{tabular}{|c|c|c|c|c|c|c|}
\hline & \multicolumn{3}{|l|}{ M2 } & \multicolumn{3}{|c|}{ Leishmania infantum } \\
\hline & Infected & Control & $\mathrm{P}$ & G1 & G2 & $\mathrm{P}$ \\
\hline Spleen & 24.4 & 3.5 & 0.0003 & 3864.0 & 706.1 & 0.0010 \\
\hline Liver & 8.0 & 7.9 & 0.3579 & 145.0 & 54.27 & 0.2312 \\
\hline Muzzle & 6.6 & 1.5 & 0.0025 & 24.0 & 98.13 & 0.6106 \\
\hline Ear & 9.2 & 1.2 & 0.0047 & 87.0 & 69.83 & 0.9823 \\
\hline Popliteal & 25.4 & 3.7 & 0.0006 & 2008.0 & 377.3 & 0.0037 \\
\hline Prescapular & 21.2 & 2.3 & 0.0036 & 1584.0 & 308.1 & 0.0049 \\
\hline
\end{tabular}

Infected = canine visceral leishmaniasis group; control=canine control groups; G1 = with skin lesions; G2 = without skin lesions; P=statistical significance. Different lowercase letters in line to each variable indicate significant differences between groups/organs. Mann-Whitney Nonparametric test to the medians M2 macrophages and for the number of DNA copies of Leishmania infantum.

the organs with highest parasitism. However, the results from the current study were discordant from those obtained by Sanchez et al. (2004) and Lima et al. (2007), since a greater parasite DNA load in the lymphoid organs than in the skin of the Leishmania infected group was detected in our study. Similarly to these findings, Moreira et al. (2013) reported higher parasitism in peripheral lymph nodes than in the skin of dogs with VL.
The higher susceptibility of the lymphoid organs to Leishmania infection was also reported by other studies confirming that these tissues are rich in mononuclear phagocyte system cells and had higher density of parasites. We attributed these characteristics to compartmentalization of specific organ immune response, as Reis et al. (2009) described by evaluating different organs of dogs with VL and finding marked differences in organ resistance or susceptibility to Leishmania infection.

Macrophages can be activated in response to the microenvironment injury, as occurred during Leishmania infection and undergo polarization to M1 or M2 phenotypes (Casseta et al., 2011) and this can be a reason for the higher number of M2 macrophages in Leishmania parasitized macrophages in most of tissue samples examined in this study, except the liver. It can be speculated that the polarization for macrophages of M2 phenotype possibly favors the survival of this parasite by creating an anti-inflammatory microenvironment that inhibits a Th1/Tc effector immune responses against Leishmania. Similar findings were reported for Post kala-azar dermal leishmaniasis, in which M2 macrophage subsets appear to be more activated, and might act to suppress cell-mediated immunity, conferring great susceptibility to intra-cellular infection and sustained disease chronicity of leishmaniasis (Mukhopadhyay et al., 2015).

Engwerda and Kaye (2000) found in a mouse model infected with L. donovani and L. infantum that different organs had specific

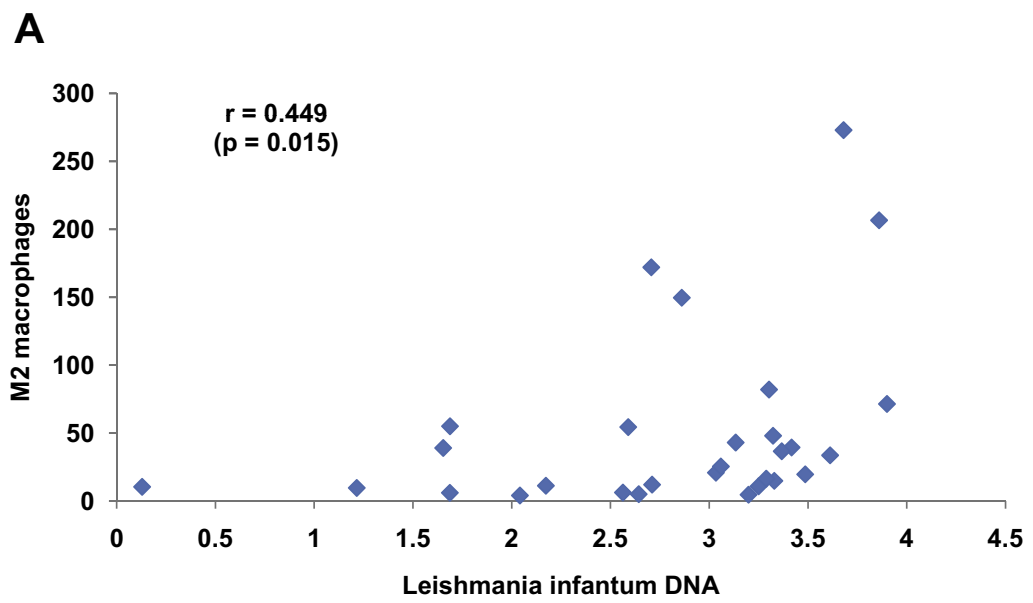

\section{B}

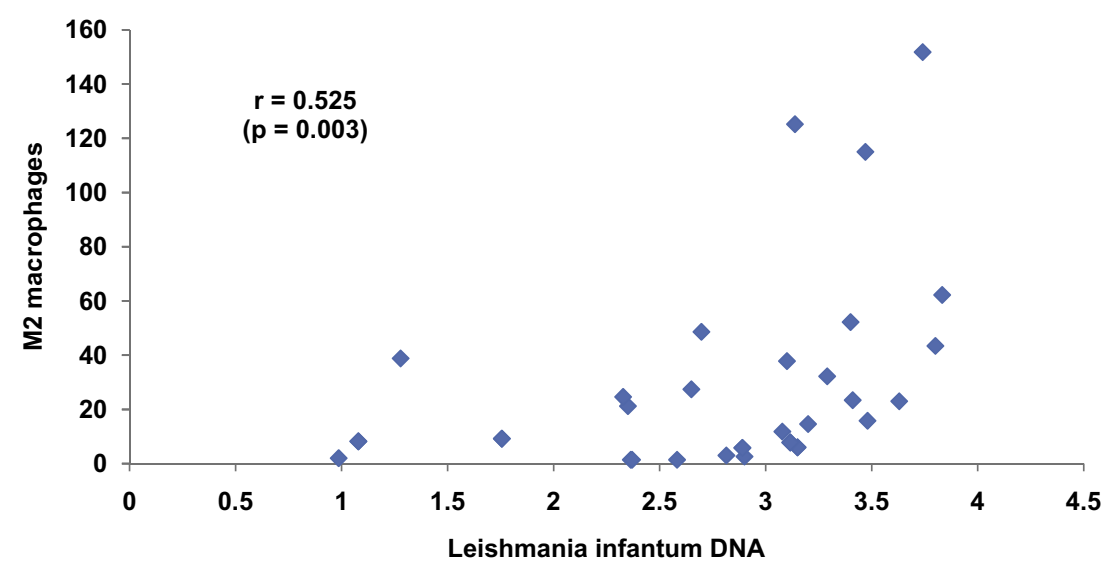

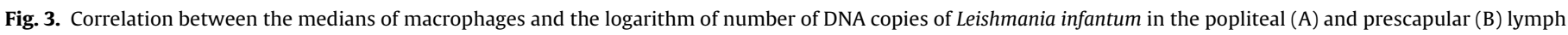
nodes of dogs from Leishmania infected group determined by linear regression analysis and the Spearman correlation coefficients $(r)$ ( $p<0.05$ ). 
responses to Leishmania infection. The liver showed increased $\mathrm{T}$ lymphocyte effector activity in these mice experimentally infected with Leishmania and this response was efficient in controlling and resolving the disease. Overall, these results were similar to those observed here in dogs with VL, demonstrating that the interaction of the parasite with the microenvironment of each organ is vital for its survival and multiplication in the host. The liver is highly efficient in pathogen clearance, probably due to its differentiated cell population, such as Kupffer cells, hepatic natural killer cells and hepatic stellate cells (Luo et al., 2000; Duarte et al., 2008). All of these cells have a close relationship with the endothelium of sinusoids and with hepatocytes, and they immediately become activated by injure stimulus, such as those mediated by Leishmania. On the other hand, the spleen and lymph nodes of naturally Leishmania infected dogs can become the ideal environment for growth of these pathogens because these parasites have a great capability of escaping from the host immunological mechanisms and can multiply intensively in these organs. In this study, these organs showed a high number of M2 macrophages and high parasite load. Additionally in the lymph nodes, as the number of parasites increased, the number of M2 macrophages increased. This can contribute to disorganization of the spleen and lymph nodes architecture induced by Leishmania-infection, due to accumulation of parasitized macrophages as reported in other studies (Tafuri et al., 1996; Lima et al., 2007; Moreira et al., 2013).

Moreover, the predominant cytokine profile secreted by Th1 or Th2 lymphocytes in the microenvironment of each Leishmania infected organ determine the polarization of macrophage phenotype and define the type of macrophage that will be activated and predominate in each phase of the Leishmania infection. This polarization might exert a control over the Leishmania infection at the initial location of the tissue injury, or in the systemic sites after dissemination of this pathogen. Thus, the skin seems to contribute towards an inefficient control over the infection and towards its visceral transformation, because when the Leishmania infection is at this location, the immune response is not effective in restrict and eliminate this parasite, due to polarization for M2 macrophages and to the inability of the M2 macrophages to destroy these parasites, or to activate an effective adaptive cell-mediated immune response. Therefore, the predominance of M2 macrophages in certain organs from naturally Leishmania infected dogs, possibly favored the parasite and contributed to the elevated parasitism in these organs. However, further studies are necessary to better elucidate this immunopathogenic process in dog Leishmaniasis.

\section{Conclusion}

The M2 macrophages seem to critically influenced the course of Leishmania infection in certain organs of dogs naturally infected that differed as to their susceptibility or resistance to the infection with this parasite. Thus, the spleen and peripheral lymph nodes presented higher susceptibility to multiplication of Leishmania, coinciding with a higher proportions of M2 macrophages in these organs, while the liver is more resistant to Leishmania infection and has a low proportion of M2 macrophages. In addition, M2 macrophages predominate, in the skin, suggesting that the local immune responses are inefficient in controlling the Leishmania infection at its initial stage, favoring therefore the progress of this infection to visceral transformation.

\section{Ethical standards}

The design for this study was approved by the Ethics and Animal Welfare Committee (CEUA n ${ }^{\circ}$. 028220/12), of FCAV/UNESP, Jaboticabal, State of São Paulo, Brazil.

\section{Conflict of interest}

The authors declare that they have no conflict of interest.

\section{Acknowledgements}

Financial assistance was provided by a grant from FAPESP (Fundação de Amparo à Pesquisa do Estado de São Paulo; n. 2013/00763-4). The authors wish to acknowledge Mrs. F.A. Ardisson for her histotechnical assistance.

\section{References}

Barbiéri, C.L., 2006. Immunology of canine leishamniasis. Parasite Immunol. 28, 329-337.

Bretagne, S., Durand, R., Olivi, M., Garin, J.F., Sulahian, A., Rivollet, D., Vidaud, M., Deniau, M., 2001. Real-time PCR as a new tool for quantifying Leishmania infantum in liver in infected mice. Clin. Diagn. Lab. Immunol. 8, 828-831.

Casseta, L., Cassol, E., Poli, G., 2011. Macrophage polarization in health and disease. ScientificWorldJournal 11, 2391-2402.

Cecílio, P., Pérez-Cabezas, B., Santarém, N., Maciel, J., Rodrigues, V., Silva, A.C., 2014. Deception and manipulation: the arms of Leishmania, a successful parasite. Front. Immunol. Microbial Immunol. 5, 1-16, article 480.

Chan, M.M., Adapala, N., Chen, C., 2012. Peroxisome proliferator-activated receptor- $\gamma$-mediated polarization of macrophages in Leishmania infection. PPAR Res. 12, 791-796.

Duarte, M.I.S., Tuon, F.F., Pagliari, C., Kauffman, M.R., Brasil, A.R., 2008. Human visceral leishmaniasis expresses Th1 pattern in situ liver lesions. J. Infect. 57 (4), 332-337.

Edin, S., Wikberg, M.L., Dahlin, A.M., Rutegard, J., Oberg, A., 2012. The distribution of macrophages with a M1 or M2 phenotype in relation to prognosis and the molecular characteristics of colorectal cancer. PLoS One 7, e47045.

Engwerda, C.R., Kaye, P.M., 2000. Organ-specific immune responses associated with infectious diseasel. Viewpoint Immunol. Today 21 (2), 73-78.

Komohara, Y., Hirahara, J., Horikawa, T., Kawamura, K., Kiyota, E., Sakashita, N., Araki, N., Takeya, M., 2006. AM-3K, an anti-macrophage antibody, recognizes CD163, a molecule associated with an anti-inflammatory macrophage phenotype. J. Histochem. Cytochem. 54 (July(7)), 763-771.

Lima, W.G., Oliveira, P.S., Caliari, M.V., Gonçalves, R., Michalick, M.S.M., Melo, M.N., Tafuri, W.L., Tafuri, W.L., 2007. Histopathological and immunohistochemical study of type 3 complement receptors (CD11b/CD18) in livers and spleens of asymptomatic and symptomatic dogs naturally infected with Leishmania (Leishmania) chagasi. Vet. Immunol. Immunopathol. 117, 129-136.

Luo, D.Z., Vermijlen, D., Ahishali, B., Triantis, V., Plakoutsi, G., Braet, F., Vanderkerken, K., Wisse, E., 2000. On the cell biology of pit cells: the liver-specific NK cells. World J. Gastroenterol. 6,1-11.

Moreira, P.R.R., Vieira, L.M., Andrade, M.M.C., Bandarra, M.B., Machado, G.F., Munari, D.P., Vasconcelos, R.O., 2010. Immune response pattern of the popliteal lymph nodes of dogs with visceral leishmaniasis. Parasitol. Res. 107, 605-613.

Moreira, P.R.R., Bandarra, M.B., Magalhães, G.M., Munari, D.P., Prandini, M.M., Alessi, A.C., Vasconcelos, R.O., 2013. Influence of apoptosis on the cutaneous and peripheral lymph node inflammatory response in dogs with visceral leishmaniasis. Vet. Parasitol. 192, 149-157.

Mukhopadhyay, D., Mukherjee1, S., Roy1, S., Dalton, J.E., Kundu1, S., Sarkar1, A. Das, N.K., Kaye, P.M., Chatterjee1, M., 2015. M2 Polarization of Monocytes-Macrophages is a hallmark of indian post kala-azar dermal leishmaniasis. PLOS Negl. Trop. Dis. 9 (10), e0004145.

Muraille, E., Leo, O., Moser, M., 2014. Th1/Th2 paradigm extended: macrophage polarization as an unappreciated pathogen-driven escape mechanism? Front. Immunol. 5, 1-12, article 603.

Oliveira, T.M.F.S., Furuta, P.I., Carvalho, D., Machado, R.Z., 2008. A study of cross-reactivity in serum samples from dogs positive for Leishmania sp, Babesia canis and Ehrlichia canis in enzyme-linked immunosorbent assay and indirect fluorescent antibody test. Rev. Bras. Parasitol. Vet. 17, 7-11.

Reis, A.B., Martins-Filho, O.A., Carvalho, A.T., Giunchetti, R.C., Carneiro, C.M., Mayrink, W., Tafuri, W.L., Oliveira, R.C., 2009. Systemic and compartmentalized immune response in canine visceral leishmaniasis. Vet. Immunol. Immunopathol. 128, 87-95.

Sanchez, M.A., Diaz, N.L., Zerpa, O., Negron, E., Convit, J., Tapia, F.J., 2004. Organ-specific immunity in canine visceral leishmaniasis: analysis of symptomatic and asymptomatic dogs naturally infected with Leishmania chagasi. Am. J. Trop. Med. Hyg. 70 (6), 618-624

Tafuri, W.L., Tafuri, W.L., Barbosa, J.A., 1996. Histopathology and immunocytochemical study of type 3 and 4 complement receptors in the liver and spleen of dogs naturally and experimentally infected with Leishmania (Leishmania) chagasi. Rev. Inst. Med. Trop. Sao Paulo 38, 81-89.

Yamate, J., Yoshida, H., Tsukamoto, Y., Ide, M., Kuwamura, M., Ohashi, F., Miyamoto, T., et al., 2000. Distribution of cells immunopositive for AM-3K, a novel monoclonal antibody recognizing human macrophages, in normal and diseased tissues of dogs cats, horses, cattle, pigs, and rabbits. Vet. Pathol. 37, $168-176$. 